\title{
The "Important Project": An Important Project of Pregnant, Obese Women
}

\author{
Wiberg-Itzel E* \\ Associate Professor, Department of Obstetrics \& \\ Gynecology, Karolinska Institute, Soder Hospital, \\ Stockholm, Sweden \\ *Corresponding author: Eva Wiberg-Itzel, Associate \\ Professor, Department of Obstetrics \& Gynecology, \\ Karolinska Institute, Soder Hospital, Stockholm, Sweden
}

Received: March 22, 2021; Accepted: April 14, 2021; Published: April 21, 2021

\section{Introduction}

Obesity has reached nearly epidemic proportions [1,2]. Today, more people die from obesity-related ailments than from starvation [3]. Since 1980, world obesity has risen from 857 million to more than two billion people, according to a recent report in the Lancet. This means that almost one in three people on earth is fat. Egypt is one of the countries in the world with the most significant number of obese people. Here are $35.9 \%$ of the population obese, according to recent figures. The U.S comes close behind in the obesity league. Nearly 78 million people are obese, which means about $33 \%$ of the population. Small countries in the Pacific are also severely affected; Countries such as Nauru, Tonga, the Cook Islands, Niue, and Samoa, where up to $50 \%$ of the population is obese. The proportion of obesity among children is also steadily increasing. According to an article in the New England Journal in 2017, 15.3\% of Chinese children are obese. The corresponding figures in the U.S. are 13\% (Table 1).

\section{Obesity Associates with Diseases}

The proportion of women with obesity in early pregnancy is continuously increasing. A high maternal BMI is associated with increased risks of complications during pregnancy, delivery, and the postpartum period. Complications for the woman and the child are described as an over-representation of fetal malformations, miscarriage, preterm birth, Intrauterine Fetal Death (IUFD), maternal hypertension, preeclampsia, and hepatosis. Other fetal complications described can be children who are Large for Gestational Age (LGA) or Small for Gestational Age (SGA). Low Apgar score at birth, respiratory disorders, and maternal and fetal postpartum infections are also associated with obesity.

In Sweden, maternity care is free, and almost all pregnant women go to a midwife during their pregnancy for check-ups. In 2018, $26.4 \%$ of Swedish women enrolled in the maternity clinics were overweighted (BMI $>=25$ ), and $15.1 \%$ of them were obese (BMI $>=30$ ). Significant regional differences are shown in Sweden. In some smaller communities in the countryside, almost half of all registered women had overweight or obese, while women in Stockholm have the lowest BMI in Sweden. 23.7\% of women were overweighted, and 10.9\% were obese. Despite the lower incidence, as many as one in four women are overweight, and one in ten women is obese in Stockholm. The figures

Table 1: Definition of obesity by WHO.
\begin{tabular}{|c|c|}
\hline BMI group* & Classification \\
\hline$<18.5$ & Underweight \\
\hline $18.5-24.9$ & Normal weight \\
\hline $25-29.9$ & Overweight \\
\hline $30-34.9$ & Obesity class I \\
\hline $35-39.9$ & Obesity class II \\
\hline$>=40$ & Obesity class III \\
\hline
\end{tabular}

*BMI: Body Mass Index $\left(\mathrm{kg} / \mathrm{m}^{2}\right)$.

in rural areas are even worse.

A woman's overweight or obesity during pregnancy poses a health risk to herself and her child. Previously performed studies have shown an increased risk of developing disabilities such as ADHD and other neuropsychiatric conditions if the mother is obese [4].

\section{"The Important Project"}

Soder Hospital in Stockholm is Sweden's largest maternity ward, with about 8,000 deliveries per year. The proportion of obese pregnant women is steadily increasing at the clinic, and about 150 women per year are delivered with a BMI $>=35$. This means women with a bodyweight often between 150-200 kg and more. This creates problems, practical as well as medical. Medical issues can involve difficult decisions as fetal monitoring during childbirth. Monitoring the fetus and the mother during labor can be a difficult challenge in really obese women. It is also known that cesarean sections can be purely lethal and a significant risk of exposing these large women to. There have been disasters on several occasions where women have cardiac arrest because she has been too long in the back position. More practical problems may be, for example, that the delivery equipment is not adapted for such large women. The instruments are too short and too small, the clothes cannot be buttoned, and the delivery beds cannot cope with the weight load of having these women as patients [5].

In England, since 2012, there has been a project called "The Specialist Weight Management Service (SWMS)," which is a result of growing public health issues related to obesity [6]. In this context, NICE guidelines for weight control before, during, and after pregnancy were conducted.

The service is aimed at pregnant women with a BMI $>=35$ and healthy lifestyle changes during pregnancy. It also actively encourages service use for up to 18 months after childbirth for the mother and her newborn child. The majority of referrals to the SWMS project are received from midwives, but many women also apply for it themselves. Pregnant women with high obesity are seen interdisciplinary at the clinic. It features a dietitian, physiotherapist, and health improvement midwife. Service users can also access 
medical consultants and health trainers if necessary. The staff uses a patient-centered behavioral change strategy and uses motivational interview techniques to help patients implement positive lifestyle changes. They also allow women to other services, including smoking cessation, breastfeeding networks, and healthy start-ups services, to name a few. This multidisciplinary approach to weight control is unique, and statistics show that support offered to pregnant women during the ante and post-natal period is a great success. This is, of course, something that should exist in every country to improve care for women with high BMI during their pregnancy, especially since this is a growing group and an explicit risk group. In many ways.

By studying the SWMS project, "The Important Project" was created at Soder hospital in Stockholm. "The Important Project" was called this because this group of women is considered "Important". The model for this project is something completely new and unique in Swedish obstetric care. The originality of the project is therefore very high. "The Important project" will handle pregnant women from different parts of Stockholm with a BMI $>=35$ when attending prenatal care. Interdisciplinary care will take place in a similar way as in the SWMS project. Various professions such as obstetricians, midwives, physiotherapists, and dietitians will be linked to the project.

A closed F.B. group will also be created with the possibility of discussion within the group and provide a faster contact with the healthcare providers. The chat feature will be available every afternoon Monday-Friday. This is an easy way to reach the responsible midwife or obstetrician and the women to exchange thoughts with each other. Women with a high BMI are a group of patients who do not seek medical care unnecessarily. Many times, they have a poor experience in healthcare and often feel mistreated.

The project was born after several women with a high BMI came to the clinic completely unprepared to give birth. This created significant problems and was also a hazardous and dangerous situation for women and her child. To create a reception where women are welcome and best looked after in an interdisciplinary way that is the "Important Project" goal. The women included in "the important project" will meet the obstetrician and midwife on at least two occasions during their pregnancy-all according to an established protocol similar to the English Nice guidelines for weight control. Analyses of blood status, vitamins, trace elements, and blood fats will be carried out. Many overweight women have a one-sided diet, and therefore more easily suffer from deficiency diseases that can create problems later in pregnancy. Decisions regarding Anticoagulants medication and possibly glucose load will be taken. Contact with a physiotherapist and a dietitian is offered.

Visit number two is prenatal planning. At that meeting, the woman+, the responsible obstetrician, + midwife meet. An appointment is also booked for the woman to meet an anesthesiologist. Contact with the midwife will also include practicalities for childbirth. The woman's wishes are collected, and, for example, suggestions for alternative birth positions are given.

A follow-up contact takes place six months after childbirth. Here a follow-up of the state of health of the woman and the child will be done, all by analogy with the SWMS project.

"The Important project" is unique in Swedish Obstetrics. Nothing like this has ever existed before. Our opinion is that women have the right to equal care regardless of their body size. She also has the right to be treated in a good and professional way. Trying to get women to lose weight during their pregnancy is not the goal of this project. Many other projects aim to do so. The "important project" wants women to feel well cared for and prepared for their pregnancy and childbirth. This benefits society, but also the woman herself.

\section{References}

1. Flegal KM, Kruszon-Moran D, Carroll MD, Fryar CD, Ogden CL. Trends in Obesity Among Adults in the United States, 2005 to 2014. Jama. 2016; 315: 2284-2291.

2. Trends in the adult body-mass index in 200 countries from 1975 to 2014: a pooled analysis of 1698 population-based measurement studies with 19.2 million participants. Lancet (London, England). 2016; 387: 1377-1396.

3. Scott-Pillai R, Spence $D$, Cardwell $C R$, et al. The impact of body mass index on maternal and neonatal outcomes: a retrospective study in the U.K. obstetric population, 2004-2011. BJOG. 2013; 120: 932-939.

4. Marchi J, Berg M, Dencker A, Olander EK, Begley C. Risks associated with obesity in pregnancy, for the mother and baby: a systematic review of reviews. Obese Rev. 2015; 16: 621-638.

5. Melchor I, Burgos J, del Campo A, Aiartzaguena A, Gutiérrez J, Melchor JC. Effect of maternal obesity on pregnancy outcomes in women delivering singleton babies: a historical cohort study. J. Perinat. Med. 2019; 47: 625630.

6. Bryant J. Weight management during and after pregnancy: A multidisciplinary approach. Pregnancy Hypertension. 2014; 4: 232. 